\title{
Considerations of physicians about the depth of palliative sedation at the end of life
}

\author{
Siebe J. Swart MD, Agnes van der Heide MD PhD, Lia van Zuylen MD PhD, Roberto S.G.M. Perez PhD, \\ Wouter W.A. Zuurmond MD PhD, Paul J. van der Maas MD PhD, Johannes J.M. van Delden MD PhD, \\ Judith A.C. Rietjens PhD
}

Competing interests: Roberto Perez has received grant funding from Hospice Kuria. Wouter Zuurmond is a board member of Nycomed, has received payment for lectures from Abott and Janssen Pharmacentre, and royalties for books on palliative care. No competing interests declared for Siebe Swart, Agnes van der Heide, Lia van Zuylen, Paul van der Maas, Johannes van Delden and Judith Rietjens.

This article has been peer reviewed.

Correspondence to:

Dr. Siebe J. Swart,

s.swart@erasmusmc.nl

CMAJ 2012. DOI:10.1503 /cmaj.110847

\begin{abstract}
Background: Although guidelines advise titration of palliative sedation at the end of life, in practice the depth of sedation can range from mild to deep. We investigated physicians' considerations about the depth of continuous sedation.

Methods: We performed a qualitative study in which 54 physicians underwent semistructured interviewing about the last patient for whom they had been responsible for providing continuous palliative sedation. We also asked about their practices and general attitudes toward sedation.

Results: We found two approaches toward the depth of continuous sedation: starting with mild sedation and only increasing the depth if necessary, and deep sedation right from the start. Physicians described similar determinants for both approaches, including titration of sedatives to the relief of refractory symptoms, patient

preferences, wishes of relatives, expert advice and esthetic consequences of the sedation. However, physicians who preferred starting with mild sedation emphasized being guided by the patient's condition and response, and physicians who preferred starting with deep sedation emphasized ensuring that relief of suffering would be maintained. Physicians who preferred each approach also expressed different perspectives about whether patient communication was important and whether waking up after sedation is started was problematic.

Interpretation: Physicians who choose either mild or deep sedation appear to be guided by the same objective of delivering sedation in proportion to the relief of refractory symptoms, as well as other needs of patients and their families. This suggests that proportionality should be seen as a multidimensional notion that can result in different approaches toward the depth of sedation.
\end{abstract}

$\mathrm{P}$ alliative sedation is considered to be an appropriate option when other treatments fail to relieve suffering in dying patients. ${ }^{1,2}$ There are important questions associated with this intervention, such as how deep the sedation must be to relieve suffering and how important it is for patients and their families for the patient to maintain a certain level of consciousness. ${ }^{1}$ In the national guidelines for the Netherlands, palliative sedation is defined as "the intentional lowering of consciousness of a patient in the last phase of life.",3,4 Sedatives can be administered intermittently or continuously, and the depth of palliative sedation can range from mild to deep. ${ }^{1.5}$

Continuous deep sedation until death is considered the most far reaching and controversial type of palliative sedation. Nevertheless, it is used frequently: comparable nationwide studies in Europe show frequencies of $2.5 \%$ to $16 \%$ of all deaths..$^{6-8}$ An important reason for continuous deep sedation being thought of as controversial is the possible association of this practice with the hastening of death, ${ }^{9-11}$ although it is also argued that palliative sedation does not shorten life when its use is restricted to the patient's last days of life. ${ }^{12,13}$ Guidelines for palliative sedation often advise physicians to titrate sedatives, ${ }^{2,3,14}$ which means that the dosages of sedatives are adjusted to the level needed for proper relief of symptoms. To date, research has predominantly focused on the indications and type of medications used for sedation. In this study, we investigated how physicians decide the depth of continuous palliative sedation and how these decisions relate to guidelines. 


\section{Methods}

\section{Participants}

This study is part of a larger project aimed at studying the practice of palliative sedation in the Netherlands after the introduction of a national guideline on palliative sedation. ${ }^{15}$ For the quantitative part of the study, we enrolled by random sampling physicians working in general practice, nursing homes and hospitals $(n=1580)$; of these, 370 reported about their most recent case of continuous palliative sedation. Frequent indications for sedation were dyspnea, pain and physical exhaustion. Details of this study are described elsewhere. ${ }^{15-17}$ Of the 370 respondents, 51 (22 general practitioners, 22 nursing home physicians and 7 clinical specialists) indicated that they were willing to participate in an additional qualitative interview. We also included the pilot interviews with one physician from each of the settings. In total, we interviewed 54 physicians (Table 1 ).

\section{Procedures}

We developed a semistructured interview scheme with open-ended questions. For each question, possible prompts were formulated. Questions related to the depth of sedation are listed in Box 1. The full interview scheme is available in Appendix 1 (www.cmaj.ca/lookup /suppl/doi:10.1503/cmaj.110847/-/DC1). To facilitate receiving answers that were as specific as possible, we included several questions pertaining to the case that the respondents had described in the quantitative questionnaire. Additional questions were asked about physicians' general sedation practices and attitudes.

We conducted interviews between October 2008 and April 2009. Participants gave consent for audiotaping, and the interviews lasted between 30 and 65 minutes. Information about the physician's age, sex, work experience and medical specialty was obtained from the original

\section{Box 1: Interview questions related to the} depth of sedation

1. Did this case concern mild or deep sedation?

2. Was the dose of the medication in this case determined by the desired depth of sedation or by the severity of symptoms? Why?

3. To what extent was it important in this case that the patient could communicate as long as possible?

4. Do you think, in general, that when using continuous sedation until death, the patients' consciousness should be preserved as long as possible? Why? questionnaire (Table 1). We ensured consistency among interviewers through the use of a semistructured interview with fixed prompts, a oneday training session about interview techniques and monthly meetings aimed at discussing findings and interim analyses. During one of the monthly meetings, the interviewers agreed that they had reached a saturation point (i.e., all relevant perspectives had been captured).

\section{Analysis}

The recordings were transcribed verbatim. We removed names and privacy-related information. We performed the analyses using the constant comparative method. The themes were independently derived from the interviews by S.J.S. and J.A.C.R. In addition, these authors compared and organized these themes in a coding tree, which was discussed several times with the rest of the authors, who have multiprofessional backgrounds and who had also read large parts of the raw material. The final coding tree, which captured all relevant themes for the purpose of this study, was agreed upon and used by S.J.S. and J.A.C.R. for coding all interviews independently. These authors discussed any differences. All authors discussed the coded fragments in depth during several meetings. During all phases of the analyses, alternative explanations of the findings were proposed and discussed to avoid potential preconceived notions.

Table 1: Characteristics of interviewed physicians

\begin{tabular}{|lccc|}
\hline & \multicolumn{2}{c}{ Practice location; no. (\%) of physicians } \\
\cline { 2 - 4 } Characteristic & $\begin{array}{c}\text { General practice } \\
n=23\end{array}$ & $\begin{array}{c}\text { Nursing homes } \\
n=23\end{array}$ & $\begin{array}{c}\text { Hospital } \\
n=8\end{array}$ \\
\hline Age, yr & $1(4)$ & $3(13)$ & $2(25)$ \\
\hline$<40$ & $9(39)$ & $7(30)$ & $4(50)$ \\
\hline $40-49$ & $11(48)$ & $11(48)$ & $2(25)$ \\
\hline $50-59$ & $2(9)$ & $2(9)$ & 0 \\
\hline$>60$ & $13(57)$ & $6(26)$ & $5(63)$ \\
\hline Sex & $10(44)$ & $17(74)$ & $3(38)$ \\
\hline Male & $2(9)$ & $8(35)$ & $2(25)$ \\
\hline Female & $9(39)$ & $7(30)$ & $4(50)$ \\
\hline Working in current specialty, yr) & $8(35)$ & $2(25)$ \\
\hline $0-9$ & $7(31)$ & 0 & 0 \\
\hline $10-19$ & $5(22)$ & $10(44)$ & $4(50)$ \\
\hline $20-29$ & $2(9)$ & $4(17)$ & $3(38)$ \\
\hline $30-39$ & $3(14)$ & & \\
\hline $\begin{array}{l}\text { Working in hospice or } \\
\text { palliative care unit }\end{array}$ & & & \\
\hline $\begin{array}{l}\text { Palliative care } \\
\text { consultant }\end{array}$ & & & \\
\hline
\end{tabular}


Quotes were selected by S.J.S. and J.A.C.R. to illustrate the arguments; the quotes were translated into English by a professional translator. Half of the quotes are case-specific and half are general.

\section{Results}

\section{Approaches for choosing the depth of sedation}

Independent of any specific approach, physicians considered it important that the effect of continuous palliative sedation be reflected in the appearance of the patient. (Box 2, quote 1). Within this context, there were two approaches described for choosing the depth of continuous sedation. Physicians either started with mild sedation and, when considered necessary, deepened it gradually $(n=$ 22 ; Box 2, quote 2) or they aimed for deep seda-

\section{Box 2: Approaches to the depth of continuous sedation}

In general, the effect of continuous palliative sedation therapy has to be reflected in the appearance of the patient

- Quote 1: You're looking for them to be peaceful. ... If someone is short of breath, that's when you really want to see that the fight is over that they're not twisting and turning, or breathing heavily, or sweating a lot. So you tend to base the dosage on the outwardly visible signs of suffering. - Clinical specialist A602

First approach: mild sedation and, when considered necessary, deepen it gradually

- Quote 2: It is usually a gradual process. When the sedation is light, the patient dozes off easily, but can still be woken. But if that's not really desirable, if you feel that the patient is still really suffering, that is when you give deeper sedation. ... But I don't think I ever opt for deep sedation right from the start. - Clinical specialist R566

Second approach: deep sedation right from the start

- Quote 3: Sedation with the aim that there was no longer any visible suffering, that the patient would no longer respond when you spoke to them. ... Deep sedation. - Clinical specialist A602

\section{Box 3: Arguments for starting with mild sedation}

\section{Alleviation of symptoms}

- Quote 1: Yes, on the basis of ... it was constantly being adjusted on the basis of symptoms. You start out on a treatment, you see its effect, see whether it is enough. If it isn't, then we adjust it. - Nursing home physician A367

- Quote 2: I always begin with light sedation to see if it is sufficient. And I then see what the patient needs, what he indicates, which depth of sedation he wants. If light sedation is enough - which is often the case, I find - it is fine with me. - General practitioner A165

\section{Enabling communication}

- Quote 3: Tailor it carefully, I'd say. So if I see that someone is very peaceful, but opens their eyes from time to time, or is able to say something if necessary, and also experiences it as pleasant, then I see no reason to say that the sedation should be deeper. - General practitioner R182

- Quote 4: Yes, it is always possible that they will wake up, you do tell them that. But many people find that very unpleasant - they tell you beforehand that they don't want it. But you can't guarantee that beforehand. - Clinical specialist P003 tion right from the start ( $n=32$; Box 2, quote 3$)$.

\section{Arguments for choosing the depth of sedation}

\section{Mild sedation}

There were two arguments for the alleviation of symptoms by starting with mild sedation and increasing the depth if necessary. Physicians who aimed for mild sedation considered a gradual approach toward the relief of refractory symptoms to be sufficient and most appropriate. These physicians generally referred to sedation as a process in which the depth of sedation is guided by the clinical condition of the patient and the patient's response to the sedatives (Box 3 , quote 1 and 2).

Physicians who aimed for mild sedation also considered communication of the patient with relatives and professional caregivers before and during sedation to be important. When adjusting the depth of sedation, they took into account the wishes of the patient and relatives about communication. Physicians also referred to the unpredictable course of continuous sedation, implying that waking up should always be taken into account. They did not consider waking up during sedation to be problematic if patients and relatives had been informed and prepared for this possibility (Box 3, quote 3 and 4).

\section{Deep sedation}

Physicians who aimed for deep sedation from the start also voiced alleviation of symptoms as their guiding principle. But they argued that, if there is an indication for continuous sedation, the symptoms causing suffering require the use of deep sedation right from the start (Box 4, quote 1 and 2). They stated that patients and relatives need to be reassured that the suffering will continue to be relieved once continuous sedation has been started. Refractory symptoms such as breathlessness, seizures and symptoms related to delirium were specifically mentioned as indications for deep sedation. Only physicians who practised in nursing homes mentioned deep continuous sedation as being appropriate for patients with end-stage dementia (e.g., to relieve refractory restlessness) (Box 4, quote 3).

In some cases, possible awakening of the patient during sedation was considered to be problematic. To reduce the chance of this happening, physicians aimed for deep sedation right from the start. A patient who wakes from continuous sedation could, according to the respondents, be suffering from the underlying disease and refractory symptoms, which should be prevented. Physicians also felt that waking up was 
problematic because unexpected awakening could give rise to agitation of the patient and distress of the relatives (Box 4, quote 4).

Physicians in general practice mostly considered waking up to be something that had to be prevented. However, those who practised in hospitals and nursing homes generally regarded it as something that could be remedied if necessary (Box 4, quote 5). Physicians sometimes made explicit agreements with or promises to the patient and relatives about the prevention of waking up during the course of continuous sedation. Whereas in all settings, the perspectives of the patient and relatives about waking up were considered, general practitioners, compared with the other physicians, more explicitly referred to the wishes of, agreements with and promises to patients and relatives (Box 4, quotes 6 and 7).

Most physicians who aimed for deep sedation considered that, once sedation was started, communication with the patient was no longer important. Instead, they stressed the importance of careful communication before the start of continuous sedation, both with the patient and family (Box 4, quote 8).

\section{Expert advice}

Both physicians who started with mild sedation and those who started with deep sedation mentioned the use of expert advice when choosing the depth of continuous sedation. This included instructions from palliative care teams, experienced colleagues or teams of specialized nurses; or medication schemes in guidelines and protocols (Box 5, quote 1). Expert advice was not always followed nor did it result in one preferred approach (Box 5, quotes 2-4).

\section{Interpretation}

We found that physicians have different approaches about the depth of continuous palliative sedation therapy. Physicians either aim for deep sedation right from the start or begin with mild sedation and deepen it gradually if needed. The arguments that the interviewed physicians used for a specific approach related to alleviation of symptoms, communication, the possibility of awakening and expert advice. The patient's clinical situation was taken into account, as were physicians' and patients' personal preferences.

The notion of proportionality is often mentioned in guidelines and debates about palliative sedation. Proportional sedation is typically thought to be sedation in which the dose is individually titrated to the relief of distress caused by refractory symptoms, ${ }^{-1}$ in this case, consciousness is reduced no more than necessary to adequately relieve suffering. ${ }^{18,19}$ Although most interviewed physicians did not use the term "proportionality," their statements suggest that proportionality is a major factor in their decisionmaking process. However, proportionality seems to be understood as being more than strictly titrating drugs to the relief of refractory symptoms. We found that physicians use a multidimensional concept of proportionality, in which other factors also play a role.

\section{Factors that contribute to proportionality}

First, the preferences of the patient contribute to proportional sedation. Patients' fear of awaken-

\section{Box 4: Arguments for deep continuous sedation from the start}

\section{Alleviation of symptoms}

- Quote 1: Yes, it's what I just said - semi-sedation isn't really an option. Someone who's so delirious [that it cannot be treated otherwise] has nothing to gain from mild sedation. - General practitioner A232

- Quote 2: Of course, the purpose of sedation is to show you that the patient no longer needs to have a hard time, no longer has to suffer unbearably. And in this particular case the patient needed so much sedation before she'd no longer respond to your voice. ... Of course, it's different each time ..., but I usually find that once you've decided on continuous sedation ... that you need so much, that the patient no longer responds to your voice. - Clinical specialist R521

- Quote 3: In this case it was deep sedation in a person with end-stage dementia. Which doesn't happen often, I have to say. But sometimes the situation's like the one with this patient, when you say to yourself, look, neither patient nor family have anything to gain when the patient is restless and short of breath. You can try out all kinds of medicines in the hope that they'll help, but you'd need such a mass of medication, while deep sedation was really all we needed to solve the problem. - Nursing home physician R320

\section{Prevention of awakening}

- Quote 4: At a certain moment it was more that the family felt that something had to be done - out of a fear that he would wake up ... that was the most important argument, I think — the family's fear. - General practitioner R59

- Quote 5: Recently we had a situation with someone ... well, we were still establishing the dose ... that after a time someone woke up again. So we adjusted the dose to get the sedation so deep that there were no more reactions to pain stimuli. - Nursing home physician R367

- Quote 6: Someone might say, "No, I want to sleep more deeply," and that's fine. And the patient can indicate beforehand what they want. Which is what we act on. If they say "Just let me keep on sleeping," that's fine. - General practitioner A165

- Quote 7: Yes, because it gives the family quite a shock, doesn't it, especially if you'd promised them that, no, he won't wake up again people have accepted that he'll just fall asleep and won't wake up; that things will quietly come to an end. So if someone does wake up, the first thing they'll think is "He's in so much pain that it woke him up." They then start to panic, as that's exactly what they didn't want. Q: And that's what you want to avoid? A: Yes. - General practitioner R218

- Quote 8: It is important that you don't leave it too late to talk things through with the patient and the family. Otherwise, of course, it will all go wrong. You'll have problems, either in the family or ... well, things will go badly. But if you bring it up on time ... Q: So it is especially the preparations ...? A: Yes. If you've done them properly, carrying it all out isn't the problem - if you're good at providing support ... well, then it's not really a problem. - General practitioner A165 
ing and their conviction that "it has been enough" codetermine the required dosage of sedatives. These preferences not only reflect the wishes of the patient, but they may also relate to inherent convictions of both patients and physicians on how the dying process under continuous sedation should evolve. The fact that waking up during continuous sedation was less often considered problematic by physicians practising in hospitals and nursing homes in our study may reflect that the quick availability and continuity of care may influence these preferences.

Second, preservation of communication is often considered important as it allows for the assessment of the indication for and the efficacy of palliative sedation. ${ }^{1}$ Our results, however, indicate that the preservation of communication is sometimes also considered to be a goal in itself. Physicians who stated that the preservation of communication was important, considered mild sedation, in general, to be proportionate sedation. In cases in which preservation of communication was not considered important anymore, the threshold for applying deeper sedation was lower. In such cases, physicians used phrases such as "everything had been said" and the patient was "ready to die." Although guidelines do not reflect the preservation of communication as an inherent point of consideration, physicians

\section{Box 5: Expert advice}

Quote 1: I rang the palliative care team, ... discussed it with them, and asked what the options were. And they said, "Well, all things considered, in this case this is a good option." And we talked about it with the family, and that's what I adopted. - General practitioner R117

Quote 2: But I wasn't very happy with the pharmacist's advice, which pretty much came down to what the palliative care team advise[d]: you know, put up the infusion and then wait until deep sleep begins. But if that's the method you use, the patient essentially lies there waiting to fall asleep. It can take an hour, which is very hard on them - it can be a very stressful hour. ... in fact, I think that's unethical. - General practitioner R100

Quote 3: We've got a protocol for it. And while I have to say that I don't know the protocol by heart, the "TT nurse"* I call in for the infusion pump - or pumps - she does know it by heart. So we discuss it - you know, things like "She's very restless: shall we take it a bit deeper?" and then we do. And once someone is sleeping very peacefully, we just let things go according to plan. So I think ... erhm ... I don't do it as often as the TT nurse ... - General practitioner A018

Quote 4: So with the last patient, we thought the dose proposed by the anesthetist was too high. But because the man was so cachectic we thought that if we gave that, it would be ... but in retrospect, it's probably what we should have done, as he woke up twice. Q: Why do you think that was? A: Well, according to the anesthetist, due to the initial dose ... that we should just have given the proposed bolus, which would eventually have achieved a better blood level. And because we didn't dare to do it, as it really isn't very nice if someone dies during the injection. - Clinical specialist P003

*A TT (technical team) nurse is a nurse working in home care, who has been trained to provide technical assistance to physicians. do take this into account when deciding the required depth of sedation.

Third, relatives' wishes are considered important. We have reported elsewhere that physicians and nurses sometimes feel pressure to start continuous sedation. ${ }^{15,16}$ The results of our study indicate that relatives' fears about the patient waking up contribute to physicians' decisionmaking.

Finally, physicians often reflect upon esthetic aspects related to depth of sedation. The appearance of the patient is expected to be peaceful after the start of continuous palliative sedation. In some cases, this leads to the goal of deep sedation from the start. In other cases, this leads to a more gradual approach that reflects "a more natural way of dying." ${ }^{20}$

\section{Comparison with other studies}

Recent publications describing guidelines for the use of sedation for patients nearing death acknowledge that repeated doses of sedatives, titrated to ease an individual's distress, are the mark of proportionate sedation..$^{1,2}$ In addition, they suggest that clinicians' considerations for using sedation must be within accepted medical guidelines of beneficence, nonmaleficence and informed consent. Differences in the frequency of the application of continuous deep sedation ${ }^{6-8}$ potentially reflect differences in attitudes toward the use and depth of sedation. Our study contributes to discussions about the proportionality of palliative sedation ${ }^{9,21}$ by adding practice-based information to theoretical and moral reasoning.

Quill and colleagues proposed that there are three types of palliative sedation: ordinary sedation, proportionate sedation and palliative sedation to unconsciousness. ${ }^{9}$ However, based on our results, we support the suggestion that all palliative sedation should be classified as proportionate sedation, ${ }^{21}$ because our results suggest that, from a multidimensional perspective on proportionality, palliative sedation to unconsciousness can be regarded as proportionate sedation in specific circumstances. The proportionality of continuous palliative sedation is determined within a context in which patients, relatives and caregivers interact and interpret clinical signs and symptoms and their consequences. This supports the notion that clinical decision-making at the end of life should be a shared deliberative process involving physicians and patients. ${ }^{22}$ Because the use of both mild and deep sedation was based on expert advice (i.e., from guidelines or palliative care experts) in our study, expert advice becomes part of this context. Apparently, its meaning and practical consequences are open to interpretation. If one interprets expert advice 
(e.g., a guideline) as suggesting that gradual deepening of sedation is the preferred approach in all cases, one could conclude that this guideline is not always followed. If, however, one accepts a multidimensional concept of proportionality as a cornerstone for decision-making about continuous sedation, then one can conclude that proportionality is indeed used for determining the depth of sedation, but in a broader sense. Others have suggested that the "proportionality principle" should be distinguished from the "principle of therapeutic responsiveness" 23 in order to differentiate physical (neurocognitive) suffering from existential agent-narrative suffering. Such an approach fits well in the multidimensional concept of proportionality which we suggest, because the principle of therapeutic responsiveness supports physicians in distinguishing between therapeutic options that are within their realm (e.g., prescribing opioids) and therapeutic options for which others have to be involved (e.g., spiritual needs). Because it has been proposed that the main objective of advance-care planning is to prepare patients and relatives to participate in making the best possible in-the-moment decision, ${ }^{24}$ it is important that the writers of guidelines pay attention to the management of expectations about the course of continuous palliative sedation from the perspective of patients, families and clinicians.

\section{Strengths and limitations}

A major strength of a qualitative study is that it allows for an in-depth analysis of arguments involved in medical decision-making. Although we did not pursue a design of purposive sampling, the large number of interviews held with respondents who had experience with continuous sedation and who were working in different settings allowed for a broad practice-based description of continuous sedation. Moreover, using each physician's most recent case as a starting point for the interviews facilitated the collection of specific information. Because this may not always be a reflection of physicians' usual practices or approaches, the most recent case served as a reference for more general reflections during the interview, which we also included in the analyses. Physicians sometimes mentioned difficulties in recollecting the details of their "most recent" patient, especially when they had been involved in the care of other patients as a consultant in palliative care. Whereas physicians were randomly selected for the original questionnaire study, the present study included physicians who volunteered to participate, which could imply that only physicians with a specific interest in the research topic responded, leading to possible selection bias.

\section{Conclusions}

When providing continuous palliative sedation, physicians may aim for deep sedation from the start or choose for a more gradual approach. In both situations, proportionality refers not only to the titration of sedatives for the relief of refractory symptoms but also to titration to patients' preferences, communication needs, wishes of relatives and esthetic consequences. This suggests that proportionality should be seen as a multidimensional notion, taking into account guidelines and external advice. For further improvement of medical care at the end of life, we recommend that the arguments that physicians use about the depth of sedation should be studied in relation to the expectations of patients and families.

\section{References}

1. de Graeff A, Dean M. Palliative sedation therapy in the last weeks of life: a literature review and recommendations for standards. J Palliat Med 2007; 10:67-85

2. Cherny NI, Radbruch L. Board of the European Association for Palliative C. European Association for Palliative Care (EAPC) recommended framework for the use of sedation in palliative care. Palliat Med 2009;23:581-93.

3. Royal Dutch Medical Association. Guideline for palliative sedation. Utrecht (Netherlands); The Association; 2009. Available: http://knmg.artsennet.nl/Diensten/knmgpublicaties /KNMGpublicatie/Guideline-for-palliative-sedation-2009.htm (accessed 2011 Apr. 12).

4. Verkerk M, van Wijlick E, Legemaate J, et al. A national guideline for palliative sedation in the Netherlands. J Pain Symptom Manage 2007;34:666-70.

5. Morita T, Tsuneto S, Shima Y. Definition of sedation for symptom relief: a systematic literature review and a proposal of operational criteria. J Pain Symptom Manage 2002;24:447-53.

6. Miccinesi G, Rietjens JA, Deliens L, et al. Continuous deep sedation: physicians' experiences in six European countries. $J$ Pain Symptom Manage 2006;31:122-9.

7. Seale C. End-of-life decisions in the UK involving medical practitioners. Palliat Med 2009;23:198-204.

8. Anquinet L, Rietjens JA, Van den Block L, et al. General practitioners' report of continuous deep sedation until death for patients dying at home: a descriptive study from Belgium. Eur $J$ Gen Pract 2011;17:5-13.

9. Quill TE, Lo B, Brock DW, et al. Last-resort options for palliative sedation. Ann Intern Med 2009;151:421-4.

10. Hasselaar JG, Verhagen SC, Vissers KC. When cancer symptoms cannot be controlled: the role of palliative sedation. Curr Opin Support Palliat Care 2009;3:14-23.

11. Rietjens JA, Buiting HM, Pasman HR, et al. Deciding about continuous deep sedation: physicians' perspectives: a focus group study. Palliat Med 2009;23:410-7.

12. Sykes N, Thorns A. Sedative use in the last week of life and the implications for end-of-life decision making. Arch Intern Med 2003;163:341-4.

13. Maltoni M, Pittureri C, Scarpi E, et al. Palliative sedation therapy does not hasten death: results from a prospective multicenter study. Ann Oncol 2009;20:1163-9.

14. Hasselaar JG, Reuzel RP, Verhagen SC, et al. Improving prescription in palliative sedation: compliance with dutch guidelines. Arch Intern Med 2007;167:1166-71.

15. Swart SJ, Brinkkemper T, Rietjens JA, et al. Physicians' and nurses' experiences with continuous palliative sedation in the Netherlands. Arch Intern Med 2010;170:1271-4.

16. Swart SJ, Rietjens JA, Brinkkemper T, et al. Palliative sedation largely in accordance with Dutch national guideline [article in Dutch]. Ned Tijdschr Geneeskd 2011;155:A2857.

17. Swart SJ, Rietjens JA, van Zuylen L et al. Continuous Palliative Sedation for Cancer and Noncancer Patients. J Pain Symptom Manage 2012;43:172-81. 
18. Claessens P, Menten J, Schotsmans P, et al. Palliative sedation: a review of the research literature. J Pain Symptom Manage 2008; 36:310-33.

19. Henry B, Dean M, Cellarius V, et al. To "sleep until death." Hastings Cent Rep 2011;41:4; author reply 4-5.

20. Raus K, Sterckx S, Mortier F. Continuous deep sedation at the end of life and the 'natural death' hypothesis. Bioethics 2011 Jan. 17 [epub ahead of print].

21. Cellarius V, Henry B. Justifying different levels of palliative sedation. Ann Intern Med 2010;152:332; author reply 333.

22. Jansen LA. Deliberative decision making and the treatment of pain. J Palliat Med 2001;4:23-30.

23. Jansen LA, Sulmasy DP. Proportionality, terminal suffering and the restorative goals of medicine. Theor Med Bioeth 2002;23: 321-37.

24. Sudore RL, Fried TR. Redefining the "planning" in advance care planning: preparing for end-of-life decision making. Ann Intern Med 2010;153:256-61.

Affiliations: From the Department of Public Health (Swart, van der Heide, van der Mass, Rietjens), Erasmus MC, Rotterdam; Laurens Antonius IJsselmonde (Swart), Rotterdam; the Department of Medical Oncology (van Zuylen), Erasmus MC, University Medical Center, Rotterdam; the Department of Anaesthesiology (Perez, Zuurmond), VU University Medical Center; Hospice Kuria (Perez), Amsterdam; the EMGO+ Institute for Health and Care Research (Perez), Amsterdam; and the Julius Centre for Health Sciences and Primary Care (van Delden), University Medical Center, Utrecht, Netherlands.

Contributors: Judith Rietjens, Agnes van der Heide, Lia van Zuylen Roberto Perez and Wouter Zuurmond designed the study. Siebe Swart, Judith Rietjens and Roberto Perez collected the interview data. Siebe Swart and Judith Rietjens had full access to all of the data in the study and take responsibility for the integrity of the data and the accuracy of the data analysis. Siebe Swart and Judith Rietjens did the primary analyses. Siebe Swart, Judith Rietjens, Agnes van der Heide, Johannes van Delden, Lia van Zuylen and Paul van der Maas contributed to the further analyses. Siebe Swart wrote the manuscript, which all authors critically reviewed and approved before submission for publication.

Funding: This study was funded by the Netherlands Organisation for Health Research and Development (ZonMw), the Sint Laurensfonds Rotterdam, Stichting Palliatieve Zorg Dirksland-Calando. The funding sources were not involved in the conduct of the study or the development of the article.

Acknowledgements: The authors thank Anneke Tooten, Tijn Brinkkemper and Gwendolyn Salvelder for interviewing respondents. 